\title{
Topological versus spectral properties of random geometric graphs
}

\author{
R. Aguilar-Sánchez ${ }^{1}$ J. A. Méndez-Bermúdez ${ }^{2,3}$, Francisco A. Rodrigues ${ }^{2}$, and José M. Sigarreta ${ }^{4}$ \\ ${ }^{1}$ Facultad de Ciencias Químicas, Benemérita Universidad Autónoma de Puebla, Puebla 72570, Mexico \\ ${ }^{2}$ Departamento de Matemática Aplicada e Estatística, \\ Instituto de Ciências Matemáticas e de Computação, \\ Universidade de São Paulo - Campus de São Carlos, \\ Caixa Postal 668, 13560-970 São Carlos, SP, Brazil \\ ${ }^{3}$ Instituto de Física, Benemérita Universidad Autónoma de Puebla, Apartado Postal J-48, Puebla 72570, Mexico \\ ${ }^{4}$ Facultad de Matemáticas, Universidad Autónoma de Guerrero, \\ Carlos E. Adame No.54 Col. Garita, Acapulco Gro. 39650, Mexico
}

\begin{abstract}
In this work we perform a detailed statistical analysis of topological and spectral properties of random geometric graphs (RGGs); a graph model used to study the structure and dynamics of complex systems embedded in a two dimensional space. RGGs, $G(n, \ell)$, consist of $n$ vertices uniformly and independently distributed on the unit square, where two vertices are connected by an edge if their Euclidian distance is less or equal than the connection radius $\ell \in[0, \sqrt{2}]$. To evaluate the topological properties of RGGs we chose two well-known topological indices, the Randić index $R(G)$ and the harmonic index $H(G)$. While we characterize the spectral and eigenvector properties of the corresponding randomly-weighted adjacency matrices by the use of random matrix theory measures: the ratio between consecutive eigenvalue spacings, the inverse participation ratios and the information or Shannon entropies $S(G)$. First, we review the scaling properties of the averaged measures, topological and spectral, on RGGs. Then we show that: (i) the averaged-scaled indices, $\langle R(G)\rangle$ and $\langle H(G)\rangle$, are highly correlated with the average number of non-isolated vertices $\left\langle V_{\times}(G)\right\rangle$; and (ii) surprisingly, the averaged-scaled Shannon entropy $\langle S(G)\rangle$ is also highly correlated with $\left\langle V_{\times}(G)\right\rangle$. Therefore, we suggest that very reliable predictions of eigenvector properties of RGGs could be made by computing topological indices.
\end{abstract}

PACS numbers: 64.60.-i, 05.45.Pq, 89.75.Hc

\section{INTRODUCTION}

Graphs have become an essential tool in nowadays complex systems modeling since they account for the underlying structure of real-world as well as synthetic systems of current interest. Depending on their building rules, graphs can be classified as regular or random: While regular graphs are constructed deterministically, random graphs follow probabilistic construction rules. There are many well-known models of random graphs. Among them, probably the most popular are: ErdösRényi random graphs, the scale-free network model of Barabási and Albert and the small-world networks of Watts and Strogatz. In these random graph models the embedding dimension is not a relevant parameter, however, in some applications it is necessary to preserve the spatial component [1] of the complex system under study. In such a case, Random Geometric Graphs (RGGs) [2, 3] have been used to study the structure and dynamics of spatially embedded complex systems.

In its original version, RGGs [4] (named as random plane networks) consider uniformly and independently distributed vertices on the plane, where vertices are linked if they are closer than a fixed distance. Later, interesting variations of the original RGGs have been studied. Among them we can mention soft RGGs (see e.g. [5, [6] and references therein), where the connection rule between vertices becomes a probabilistic function, and random rectangular graphs 7], which were introduced as a generalization of RGGs to allow for the flex- ibility of the embedding space. Moreover, RGGs have found applications in the study of social [8] and neural networks 9], synchronization phenomena 10], wireless ad-hoc communications [12 15 and disease dynamics 16, 17. Many properties of RGGs and its variations are already known. Topological properties such as the average degree, the degree distribution, the average path length and the clustering coefficient were reported in [7]. The effects of geometrical boundaries on RGGs were aso discussed in [11]. In addition, some spectral properties of RGGs have already been reported, both theoretically [18] and numerically [18 21].

In this work, within a random-matrix-theory (RMT) approach, we perform a statistical study of both topological and spectral properties of RRGs. In particular, to evaluate the topological properties of RGGs we chose two well-known topological indices, the Randić index and the harmonic index. While we characterize the spectral and eigenvector properties of the corresponding randomly-weighted adjacency matrices by the use of RMT measures: the ratio between consecutive eigenvalue spacings, the inverse participation ratios and the information or Shannon entropies. Moreover, we report an unexpected high correlation between topological indices and the Shannon entropies of eigenvectors; thus, suggesting that very reliable predictions of eigenvector properties of RGGs could be made by just computing topological indices. 


\section{A. The randomly-weighted graph model}

We consider RGGs $G(n, \ell)$ consisting of $n$ vertices uniformly and independently distributed on the unit square, where two vertices are connected by an edge if their Euclidian distance is less or equal than the connection radius $\ell \in[0, \sqrt{2}]$. Here we will follow a recently introduced approach under which the adjacency matrices of random graphs are represented by RMT ensembles; see the application of this approach on Erdös-Rényi graphs 22], random rectangular graphs [21], $\beta$-skeleton graphs [23], multiplex and multilayer networks [24], and bipartite graphs [25]. Accordingly, we define the elements of the adjacency matrix $\mathbf{A}$ of the RGGs, $G(n, \ell)$, as

$A_{i j}=\left\{\begin{array}{cl}\sqrt{2} \epsilon_{i i} & \text { for } i=j \\ \epsilon_{i j} & \text { if there is an edge between vertices } i \text { and } j \\ 0 & \text { otherwise. }\end{array}\right.$

We choose $\epsilon_{i j}$ as statistically-independent random variables drawn from a normal distribution with zero mean and unity variance. Also, $\epsilon_{i j}=\epsilon_{j i}$, since $G$ is assumed as undirected. According to this definition, diagonal random matrices are obtained for $\ell=0$ (Poisson ensemble (PE), in RMT terms), whereas the Gaussian Orthogonal Ensemble (GOE) (i.e. full real and symmetric random matrices) is recovered when $\ell=\sqrt{2}$. Therefore, a transition from the PE to the GOE can be observed by increasing $\ell$ from zero to $\sqrt{2}$, for any given graph size $n$.

We stress that the random weights we impose to the adjacency matrix $\mathbf{A}$, as defined in Eq. (1), do not play any role in the computation of vertex-degree-based indices, however they help us obtaining non-null adjacency matrices (that we can still diagonalize) for graphs with isolated vertices; so we can safely explore spectral and eigenvector properties in the limit $\ell \rightarrow 0$. Moreover, including random weights to the standard RGG model allows us to retrieve well known random matrices in the appropriate limits in order to use RMT results as a reference.

\section{MEASURES}

\section{A. Topological measures}

Topological indices based on end-vertex degrees of edges have been used for more than 40 years and some of them are recognized tools in chemical research. Probably, the best known among such descriptors are the Randić connectivity index and the Zagreb indices.

Given a simple connected graph $G=(V(G), E(G))$ with the vertex set $V(G)$ and the edge set $E(G)$, the Randić connectivity index was defined in [26] as

$$
R(G)=\sum_{u v \in E(G)} \frac{1}{\sqrt{d_{u} d_{v}}}
$$

where $u v$ denotes the edge of the graph $G$ connecting the vertices $u$ and $v$, and $d_{u}$ is the degree of the vertex $u$. There are hundreds of papers and a couple of books dealing with $R(G)$ (see, e.g., [27-29] and the references therein). In addition to the multiple applications of the Randić index in physical chemistry, this index has found several applications in other research areas and topics, such as information theory 30, network similarity 31, protein alignment 32, network heterogeneity 33], and network robustness 34]. Moreover, in 35] the concept of graph entropy for weighted graphs was introduced, especially the Randic weights.

Other index that has attracted great interest in the last years is the harmonic index. It is given by [36]

$$
H(G)=\sum_{u v \in E(G)} \frac{2}{d_{u}+d_{v}}
$$

See examples of recent studies of $H(G)$ in Refs. 37 42 .

From definitions (2), when $\ell=0$ (i.e. when all vertices in the RGG are isolated) we have $R(G)=0$ and $H(G)=0$, while for $\ell=\sqrt{2}$ (i.e. when the RGG graph is complete), $R(G)=n / 2$ and $H(G)=n / 2$. That is, we expect to observe a transition from 0 to $n / 2$ for the quantities $\langle R(G)\rangle$ and $\langle H(G)\rangle$ when increasing $\ell$ from 0 to $\sqrt{2}$.

We want to stress that the statistical study of topological indices we perform here is justified by the random nature of the RGGs. Since a given parameter pair $(n, \ell)$ represents an infinite-size ensemble of random graphs, the computation of a topological index on a single graph is irrelevant. In contrast, the computation of a given topological index on a large ensemble of random graphs, all characterized by the same parameter pair $(n, \ell)$, may provide useful average information about the full ensemble. This statistical approach, well known in RMT studies, is not widespread in studies of topological indices, mainly because topological indices are not commonly applied to random graphs; for recent exceptions see [42, 43].

\section{B. Spectral measures}

In this work we characterize the spectral and eigenvector properties of the randomly-weighted adjacency matrices of Eq. (1) by the use of well-known RMT measures: the ratio between consecutive eigenvalue spacings $r$, the inverse participation ratios IPR and the information or Shannon entropies $S$.

On the one hand, given the ordered spectra $\left\{\lambda_{i}\right\}$, the ratio $r_{i}$ is given by 44 .

$$
r_{i}=\frac{\min \left(\lambda_{i+1}-\lambda_{i}, \lambda_{i}-\lambda_{i-1}\right)}{\max \left(\lambda_{i+1}-\lambda_{i}, \lambda_{i}-\lambda_{i-1}\right)},
$$

$i=2, \ldots, n-1$. It is known that $\langle r\rangle_{\mathrm{PE}} \approx 0.3863$, while $\langle r\rangle_{\mathrm{GOE}} \approx 0.5359$ [44]. Here and below $\langle\cdot\rangle$ denotes the average over all eigenvalues/eigenvectors of an ensemble 
of adjacency matrices $\mathbf{A}$. On the other hand, for the normalized eigenvector $\Psi^{i}$, i.e. $\sum_{j=1}^{n}\left|\Psi_{j}^{i}\right|^{2}=1$, we have

$$
\mathrm{IPR}_{i}=\left[\sum_{j=1}^{n}\left|\Psi_{j}^{i}\right|^{4}\right]^{-1}
$$

and

$$
S_{i}=-\sum_{j=1}^{n}\left|\Psi_{j}^{i}\right|^{2} \ln \left|\Psi_{j}^{i}\right|^{2}
$$

It is fair to mention that both IPR and $S$, which quantify the extension of eigenvectors in a given basis, have been widely used to study the localization characteristics of the eigenvectors of random graphs and network models. Among the vast amount of studies available in the literature, as examples of recent studies were the IPR and $S$ were applied on graphs studies, we can mention that: (i) the IPR facilitated the introduction of the concept of layer localization in multilayer random networks, this new concept was shown to have relevant implications in the dynamics of desease contagion in multiplex systems [45, 46]; also (ii) the IPR allowed to demonstrate that the eigenvectors of random networks with power-law decaying bond strengths are multifractal objects [4]; while (iii) $S$ was used to define universal parameters able to scale the eigenvector properties of multiplex and multilayer networks [24] and bipartite graphs 25]. In contrast, $r$ has been scarcely used in graph studies; for a recent exception see Ref. [48], were $P(r)$ served to characterize the percolation transition in weighted Erdös-Rényi graphs. We believe that the lack of use of $r$ in graph studies is mainly due to the fact that the introduction of $r$ is relatively recent. In fact, most studies of spectral properties of random graphs, from a RMT point of view, are based on the nearest-neighbor energy level spacing distribution $P(s)$, see e.g. 22 and the references therein. However, we emphasize that we prefer to use $\langle r\rangle$, instead of $P(s)$, because the calculation of the ratios $r_{i} \equiv \min \left(s_{i}, s_{i+1}\right) / \max \left(s_{i}, s_{i+1}\right)$ (with $s_{i}=\left(\lambda_{i+1}-\lambda_{i}\right) / \Delta, \Delta$ being the mean eigenvalue spacing $)$ do not require the spectrum unfolding, a task that may become cumbersome. Moreover, the spectrum unfolding fixes $\langle s\rangle=1$ and forbids the use of $\langle s\rangle$ as a complexity indicator; a restriction not applicable to $\langle r\rangle$.

With definitions (5 6), when $\ell=0$, since the eigenvectors of $\mathbf{A}$ have only one nonvanishing component with magnitude equal to one, $\mathrm{IPR}_{i}=1$ and $S_{i}=0$, so that $\langle\operatorname{IPR}(G)\rangle=\operatorname{IPR}_{\mathrm{PE}}=1$ and $\langle S(G)\rangle=S_{\mathrm{PE}}=0$, respectively. In the opposite limit, $\ell=\sqrt{2}$, the fully chaotic eigenvectors extend over the $n$ available vertices of $G$, so $\langle\operatorname{IPR}(G)\rangle=\operatorname{IPR}_{\mathrm{GOE}}$ and $\langle S(G)\rangle=S_{\mathrm{GOE}}$. Here, $\mathrm{IPR}_{\mathrm{GOE}} \approx n / 3$ and $S_{\mathrm{GOE}} \approx \ln (n / 2.07)$ correspond to random eigenvectors with Gaussian distributed amplitudes; i.e. eigenvectors of the GOE. Thus, we expect to observe a transition from the PE to the GOE limits for $\langle r\rangle,\langle\operatorname{IPR}(G)\rangle$ and $\langle S\rangle$ when increasing $\ell$ from 0 to $\sqrt{2}$.

\section{SCALING OF TOPOLOGICAL AND SPECTRAL MEASURES}

\section{A. Topological measures on RGGs}

Recall that increasing $\ell$ from 0 to $\sqrt{2}$ drives the RGGs from isolated vertices to complete graphs. Indeed, the set of non-isolated vertices of the RGGs, $V_{\times}(G) \subseteq V(G)$, are those that contribute to the sums in Eqs. (2) 3). Clearly, $V_{\times}(G)=0$ for $\ell=0$ while $V_{\times}(G)=n$ for $\ell=\sqrt{2}$, however the quantity $V_{\times}(G)$ as a function of $\ell$ is unknown (to the best of our knowledge) for RGGs. Therefore, together with $R(G)$ and $H(G)$, below we also compute $V_{\times}(G)$.

Now, in Figs. 11(a-c) we present $\left\langle V_{\times}(G)\right\rangle,\langle R(G)\rangle$, and $\langle H(G)\rangle$ as a function of the connection radius $\ell$ for RGGs of size $n$. All averages are computed over ensembles of $10^{7} / n$ RGGs characterized by the parameter pair $(n, \ell)$. Each panel displays four curves corresponding to different graph sizes: 125, 250, 500 and 1000 . Note that all curves $\langle X(G)\rangle$ vs. $\ell$ in Figs. $1($ a-c) display the transition (in fact, a smooth transition in semi-log scale) from isolated vertices to complete graphs when increasing $\ell$. In this Section, $X$ stands for $V_{\times}, R$ and $H$.

Given the similar functional form of the curves $\langle X(G)\rangle$ vs. $\ell$ for different graph sizes $n$, as reported in Figs. 1(ac), it seems that they could be effectively scaled. That is, one should be able to find a scaling parameter $\xi \equiv \xi(n, \ell)$ such that the curves $\langle\bar{X}(G)\rangle$ vs. $\xi$ are invariant, where $\bar{X}$ is the properly normalized measure $X$. We choose to normalize the topological indices $X$ to their maximum values: $\max \left[V_{\times}(G)\right]=n, \max [R(G)]=n / 2$ and $\max [H(G)]=n / 2$. Then, in Figs. $1(\mathrm{~d}-\mathrm{f})$ we present again $\langle X(G)\rangle$ but now normalized to $\max [X(G)]$. From these figures we can clearly see that when changing $n$ the curves $\langle\bar{X}(G)\rangle=\langle X(G)\rangle / \max [X(G)]$ keep their functional form but they suffer a displacement on the $\ell$-axis. Therefore, in order to search for the scaling parameter $\xi \equiv \xi(n, \ell)$ we first establish a quantity to characterize the position of the curves $\langle\bar{X}(G)\rangle$ on the $\ell$-axis. Since all curves $\langle\bar{X}(G)\rangle$ vs. $\ell$ transit from zero (isolated vertices) to one (complete graphs) when $\ell$ increases from zero to

TABLE I: Values of the parameters $\mathcal{C}$ and $\gamma$ obtained from the fittings of Eq. (7) to the data $\ell^{*}$ vs. $n$ of the insets in panels (d-f) of Figs. 1 and 3 .

\begin{tabular}{lll}
\hline measure & $\mathcal{C}$ & $\gamma$ \\
\hline \hline$\left\langle V_{\times}(G)\right\rangle / n$ & 0.49794 & 0.50751 \\
\hline$\langle R(G)\rangle /(n / 2)$ & 0.50519 & 0.50756 \\
\hline$\langle H(G)\rangle /(n / 2)$ & 0.51253 & 0.50764 \\
\hline$\langle\bar{r}(G)\rangle$ & 1.2883 & 0.44193 \\
\hline$\langle\overline{I P R}(G)\rangle$ & 1.1844 & 0.36272 \\
\hline$\langle S(G)\rangle / S_{\mathrm{GOE}}$ & 1.0463 & 0.44138 \\
\hline
\end{tabular}



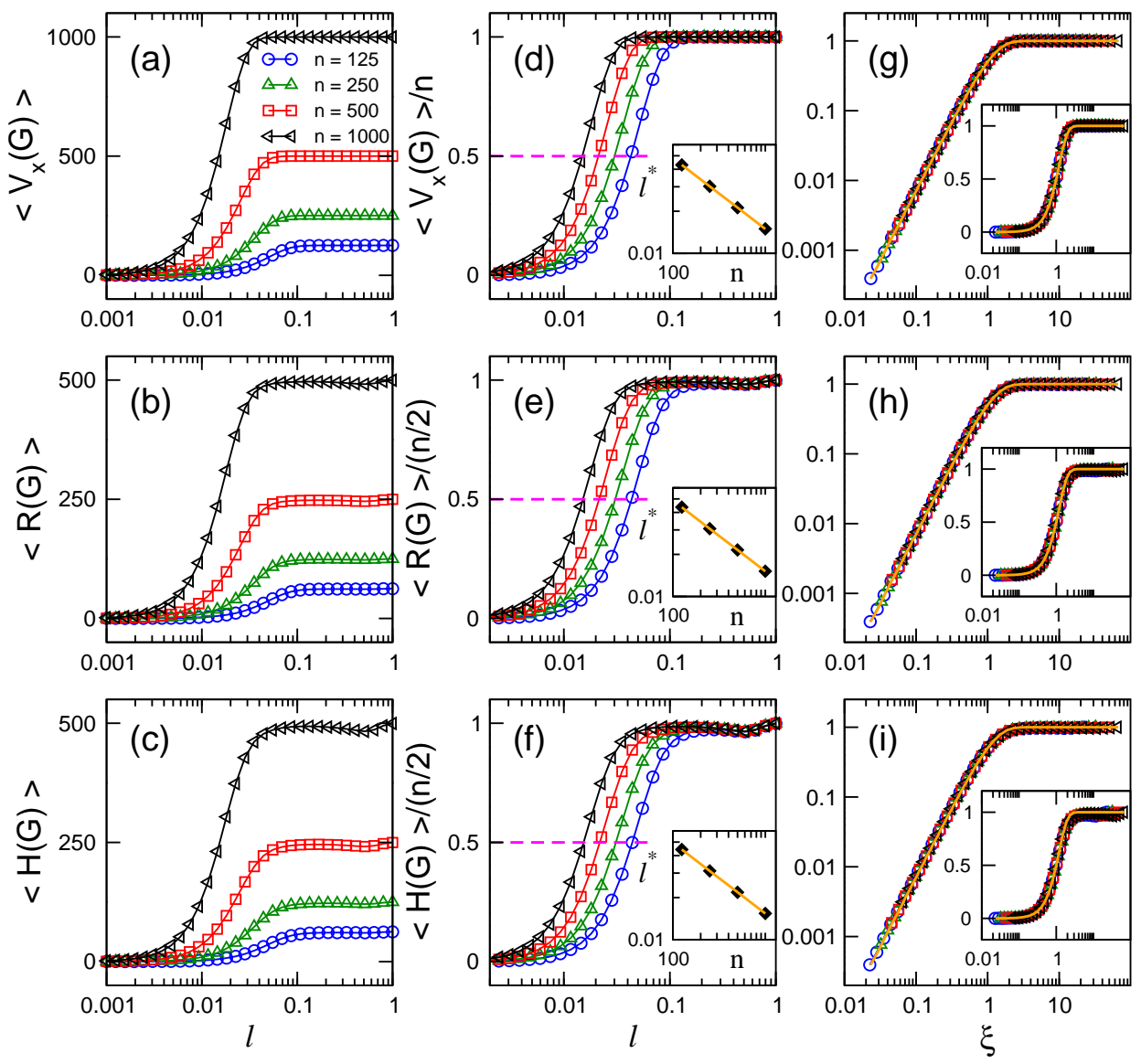

FIG. 1: (a) Average number of non-isolated vertices $\left\langle V_{\times}(G)\right\rangle$, (b) average Randić index $\langle R(G)\rangle$, and (c) average harmonic index $\langle H(G)\rangle$ as a function of the connection radius $\ell$ of random geometric graphs of four different sizes $n$. Normalized average indices $\left\langle V_{\times}(G)\right\rangle / n,\langle R(G)\rangle /(n / 2)$ and $\langle H(G)\rangle /(n / 2)$ as a function of (d-f) $\ell$ and (g-i) $\xi$. The horizontal dashed line in panels (d-f) marks $\langle\bar{X}(G)\rangle=0.5$. The insets in panels (d-f) show $\ell^{*}$ vs. $n$; the orange lines represent the best fittings of the data with Eq. (7), with fitting parameters reported in Table \ The insets in panels (g-i) show the same curves of the main panels but in semi-log scale. The orange line, shown for comparison purposes in the main panels (g-i) and the insets, is the curve $\left\langle V_{\times}(G)\right\rangle / n$ vs. $\xi$.

$\sqrt{2}$, we choose the value of $\ell$ for which $\langle\bar{X}(G)\rangle \approx 0.5$; see the horizontal dashed lines in Figs. 1(d-f). We label the value of $\ell$ at half of the transition as $\ell^{*}$.

In the insets of Figs. 1(d-f) we present the values of $\ell^{*}$, extracted from the curves of the main panels, versus $n$. Indeed, the linear trend of the data $\ell^{*}$ vs. $n$ (in log-log scale) suggests the power-law behavior

$$
\ell^{*}=\mathcal{C} n^{-\gamma} .
$$

As shown in the insets of Figs. 1(d-f), Eq. (7) provides excellent fittings to the data; see the full-orange lines. From the fitted parameters, reported in Table I. we can conclude that $\gamma \approx 1 / 2$ for the three indices: $V_{\times}(G), R(G)$ and $H(G)$.

Finally, we define the scaling parameter $\xi$ as the ratio between $\ell$ and $\ell^{*}$, so we get

$$
\xi \equiv \frac{\ell}{\ell^{*}}=\frac{\ell}{\mathcal{C} n^{-\gamma}}=\frac{n^{\gamma} \ell}{\mathcal{C}} .
$$

Therefore, by plotting again the curves of Figs. प(d-f) now as a function of $\xi$ we observe that curves for different graph sizes $n$ collapse on top of universal curves; see Figs. 1(g-i) where we present $\langle X(G)\rangle / \max [X(G)]$ vs. $\xi$ in $\log$-log scale (main panels) as well as in semi$\log$ scale (insets). Also note that the three indices are characterized by very similar universal curves. Indeed, in Figs. 1(h) and 1(i) we plot the curve $\left\langle V_{\times}\right\rangle / n$ vs. $\xi$ on top of the data for $\langle R(G)\rangle$ and $\langle H(G)\rangle$, respectively, and observe a remarkably good coincidence anticipating a very high correlation among these topological indices. Moreover, in Fig. 2 we present scatter plots between the (normalized and scaled) indices reported in Fig. 1 where the high correlation between them is evident. To quantify the correlation among these indices, in the panels of Fig. 2 we report the corresponding Pearson's correlation coefficient $\rho$, which turns out to be approximately equal to one in all cases. We note that we compute $\rho$ for the $\log$ of the variables to avoid an overestimation caused by 

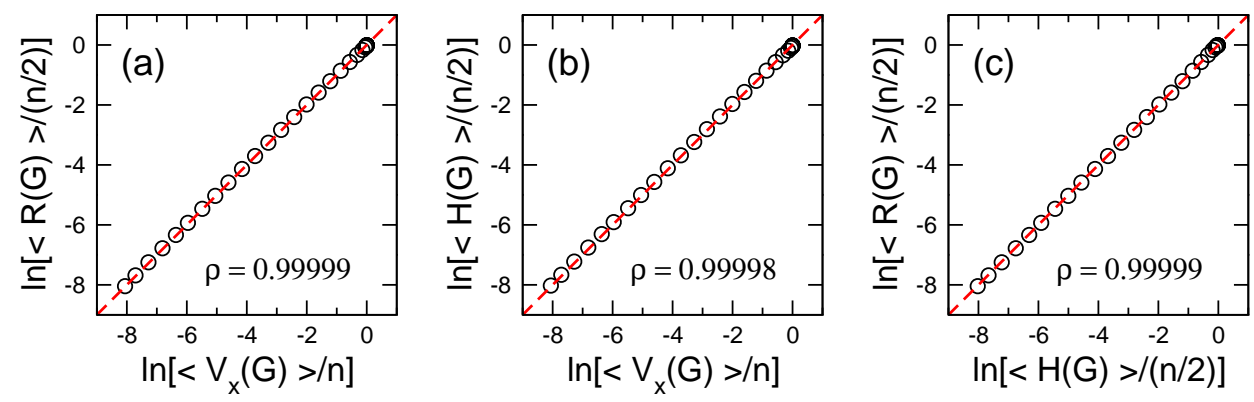

FIG. 2: Scatter plots of the log of the normalized indices reported in Fig. 1. The red-dashed lines, shown as a reference, are the identity function. The Pearson correlation coefficients are reported in the corresponding panels.

the data with $\langle\bar{X}(G)\rangle \sim 1$.

Figure 2 provides clear evidence of the relevance of the non-isolated vertices $V_{\times}(G)$ in the computation of topological indices, particularly in the computation of $R(G)$ and $H(G)$. That is, once knowing the value of the parameter $\mathcal{C}$, one could compute $\left\langle V_{\times}(G)\right\rangle$ and make quite precise predictions of $\langle R(G)\rangle$ or $\langle H(G)\rangle$ in RGGs.

\section{B. Spectral measures on RGGs}

Now we perform a scaling study of spectral and eigenvector measures of RGGs equivalent to the one made in the previous Section for topological indices. We use exact numerical diagonalization to obtain the eigenvalues $\lambda_{i}$ and eigenvectors $\Psi^{i}(i=1, \ldots, n)$ of large ensembles of adjacency matrices given by Eq. (11) (characterized by $n$ and $\ell$ ) and compute the average values of the measures of Eqs. (446).

In Figs. [3(a-c) we present $\langle r(G)\rangle,\langle\operatorname{IPR}(G)\rangle$ and $\langle S(G)\rangle$ as a function of the connection radius $\ell$ for RGGs of size $n$. All averages are computed over all eigenvalues or eigenvectors of ensembles of $10^{7} / n$ RGGs. Note that all curves $\langle X(G)\rangle$ vs. $\ell$ in Figs. 3(a-c) display the transition from the $\mathrm{PE}$ regime (isolated vertices; i.e diagonal random matrices) to the GOE regime (complete graphs; i.e full random matrices) for increasing $\ell$. In this Section $X$ stands for $r$, IPR and $S$.

To perform the scaling analysis of the curves $\langle X\rangle$ vs. $\ell$ we first normalize them. However, since $\langle r(G)\rangle \rightarrow$ const. $\neq 0$ and $\langle\operatorname{IPR}(G)\rangle \rightarrow$ const. $\neq 0$ for $\ell \rightarrow 0$, we conveniently define the corresponding normalized averages as

$$
\langle\bar{r}(G)\rangle \equiv \frac{\langle r(G)\rangle-\langle r\rangle_{\mathrm{PE}}}{\langle r\rangle_{\mathrm{GOE}}-\langle r\rangle_{\mathrm{PE}}}
$$

and

$$
\langle\overline{\mathrm{IPR}}(G)\rangle \equiv \frac{\langle\operatorname{IPR}(G)\rangle-\mathrm{IPR}_{\mathrm{PE}}}{\mathrm{IPR}_{\mathrm{GOE}}-\mathrm{IPR}_{\mathrm{PE}}} .
$$

While we normalize $\langle S(G)\rangle$ with $S_{\mathrm{GOE}}$ : $\langle\bar{S}(G)\rangle=$ $\langle S(G)\rangle / S_{\mathrm{GOE}}$. Then, in Figs. 3 (d-f) we present the normalized measures $\langle\bar{X}(G)\rangle$ as a function of $\ell$. From these figures we can see that when increasing $n$, the curves $\langle\bar{X}(G)\rangle$ keep their functional form but suffer a displacement to the left on the $\ell$-axis. Also, since all curves $\langle\bar{X}(G)\rangle$ vs. $\ell$ transit from zero (PE regime) to one (GOE regime) when $\ell$ increases from zero to $\sqrt{2}$, as in the previous Section we can use the value of $\ell$ for which $\langle\bar{X}(G)\rangle \approx 0.5, \ell^{*}$, to characterize the position of the curves $\langle\bar{X}(G)\rangle$ vs. $\ell$ on the $\ell$-axis. Notice that $\ell^{*}$ characterizes the PE-to-GOE transition of the RGGs.

In the insets of Figs. 3(d-f) we plot the values of $\ell^{*}$, extracted from the curves of the main panels, versus $n$. Again as for the topological indices, the linear trend of the data $\ell^{*}$ vs. $n$ (in log-log scale) suggests the powerlaw behavior of Eq. (7). Therefore we use Eq. (7) to fit the data in the insets of Figs. 3(d-f), obtaining excellent fittings. The fitted parameters are reported in Table \. Here, in contrast to the topological indices studied in the previous Section, we do not find the same power law $\gamma$ for all spectral measures: i.e. $\gamma \approx 0.44$ for $r(G)$ and $S(G)$, while $\gamma \approx 0.36$ for $\operatorname{IPR}(G)$. Anyway, to scale the spectral measures on RGGs we use the scaling parameter $\xi$, as defined in Eq. (8).

Thus, in Figs. 3(g-i) we plot again the curves of Figs. 3(d-f) but now as a function of $\xi$. It is interesting to note that, even though we observe a perfect collapse of the curves $\langle\bar{r}(G)\rangle$ vs. $\xi$ and $\langle S(G)\rangle / S_{\mathrm{GOE}}$ vs. $\xi$ (except for large fluctuations in the curves of $\langle\bar{r}(G)\rangle$ vs. $\xi$ for small $n$ and small $\ell$ ), the average inverse participation ratio does not scale properly for $\ell<0.5$; of course this effect is only visible in the log-log scale (see the insets where the scaling looks reasonably good).

Also, notice from Figs. 3(g) and [3(i) that the universal curves characterizing $\langle\bar{r}(G)\rangle$ and $\langle S(G)\rangle / S_{\mathrm{GOE}}$ are clearly different. Moreover, it results quite remarkable for us to observe that the universal curve of $\langle S(G)\rangle / S_{\mathrm{GOE}}$ looks similar to the universal curves of the topological indices reported in the previous Section. Indeed, in Figs. 3(h-i) we plot the curve $\left\langle V_{\times}(G)\right\rangle / n$ vs. $\xi$ on top of the data for the spectral and eigenvector measures and observe a very good coincidence with the scaled Shannon entropy. Also, in Fig. 3 we present scatter plots between the normalized measures reported in Fig. 3 and 

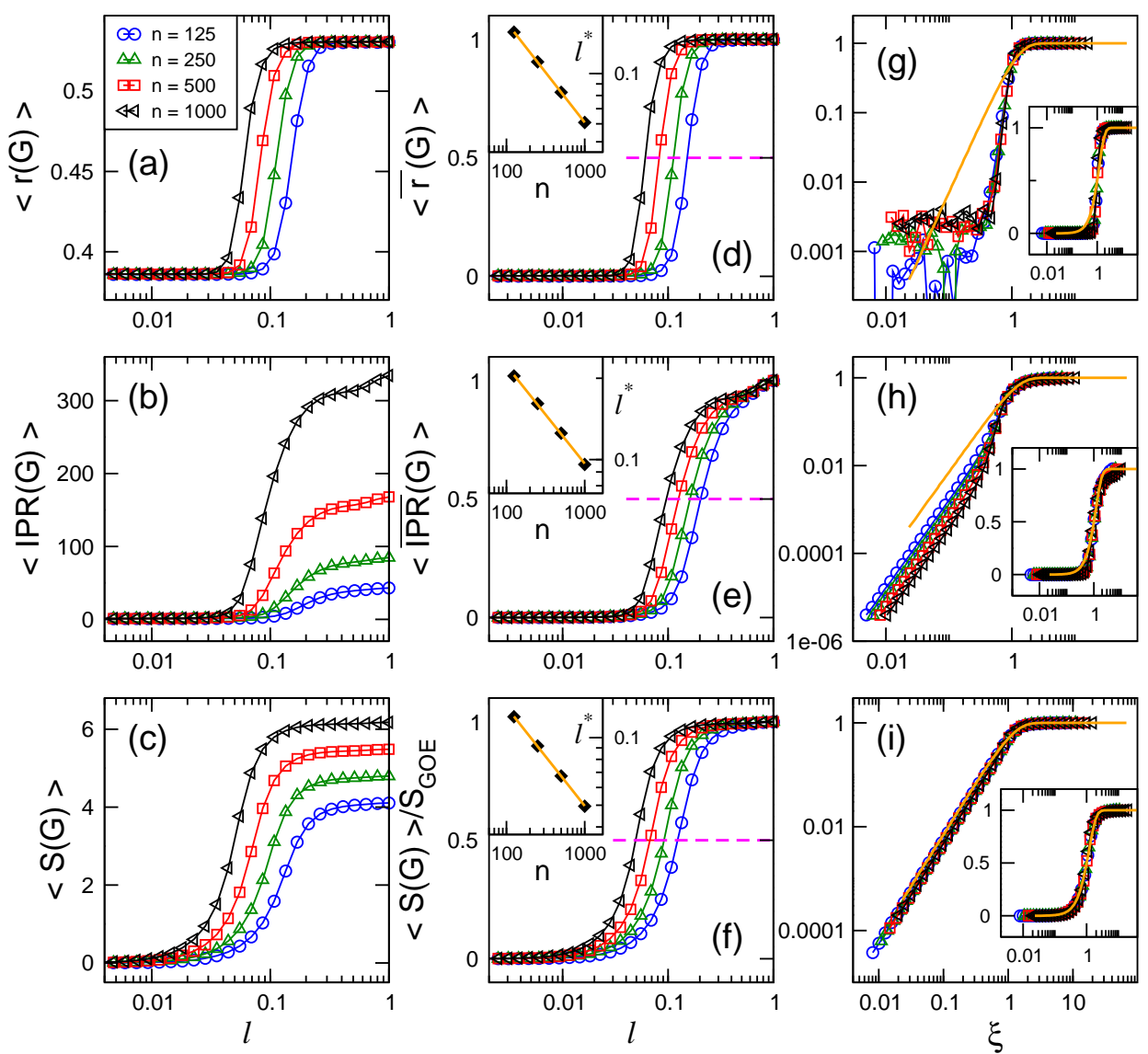

FIG. 3: (a) Average ratio between consecutive eigenvalue spacings $\langle r(G)\rangle$, (b) average inverse participation ratio $\langle\operatorname{IPR}(G)\rangle$, and (c) average Shannon entropy $\langle S(G)\rangle$ as a function of the connection radius $\ell$ of random geometric graphs of four different sizes $n$. Normalized average measures $\langle\bar{r}(G)\rangle,\langle\overline{\mathrm{IPR}}(G)\rangle$ and $\langle S(G)\rangle / S_{\mathrm{GOE}}$ as a function of (d-f) $\ell$ and (g-i) $\xi$. The insets in panels (d-f) show $\ell^{*}$ vs. $n$; the orange lines represent the best fittings of the data with Eq. (77), with fitting parameters reported in Table [. The horizontal dashed line in panels (d-f) marks $\langle\bar{X}(G)\rangle=0.5$. The insets in panels (g-i) show the same curves of the main panels but in semi-log scale. The orange line, shown for comparison purposes in the main panels (g-i) and the insets, is the curve $\left\langle V_{\times}(G)\right\rangle / n$ vs. $\xi$.
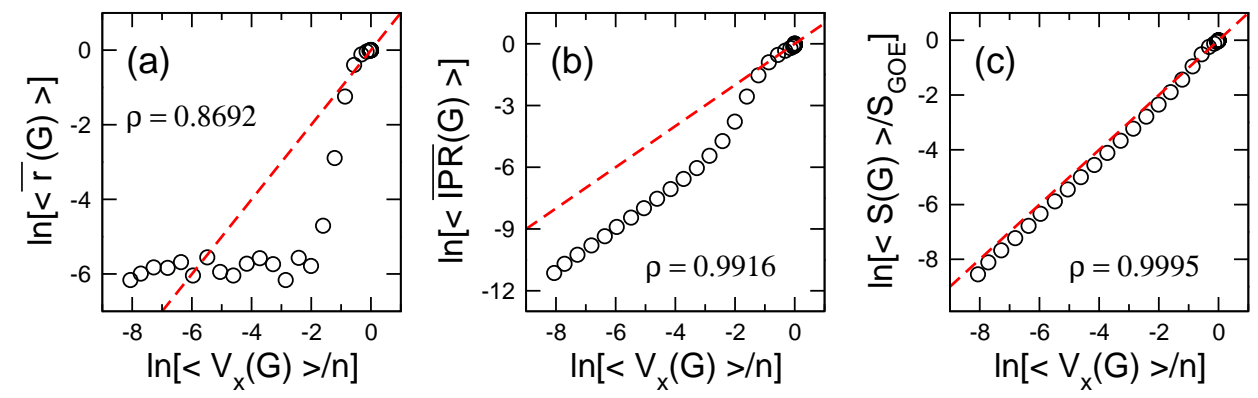

FIG. 4: Scatter plots of the log of the normalized spectral measures reported in Fig. 3 versus the log of the normalized average number of non-isolated vertices $\left\langle V_{\times}(G)\right\rangle / n$. The red-dashed lines, shown as a reference, are the identity function. The Pearson correlation coefficients are reported in the corresponding panels.

the scaled average number of non-isolated vertices. There the correlation between $S(G)$ and $V_{\times}(G)$ is evident, with a Pearson's correlation coefficient $\rho \approx 1$.

\section{CONCLUSIONS}

In this work we unveil an important link between topological and eigenvector properties of random geomet- 
ric graphs (RGGs). Specifically, we numerically show that the Shannon entropies $S(G)$ of the eigenvectors of the randomly-weighted adjacency matrices of RGGs are highly correlated with the number of non-isolated graph vertices $V_{\times}(G)$ as well as with the Randić index $R(G)$ and the harmonic index $H(G)$. Thus, we suggest that reliable predictions of eigenvector properties of RGGs could be made by computing topological indices. Clearly, an open question we plan to address in a future investigation is whether the correlation between topological indices and Shannon entropies also appears in other random network models.

Recently, it was demonstrated that the parameter that scales a large variety of average topological indices, including $R(G)$ and $H(G)$, on random graphs is the average degree [49]; which for RGGs is given by [7] $\langle k\rangle=$ $(n-1)\left(\pi \ell^{2}-8 \ell^{3} / 3+\ell^{4} / 2\right)$. Instead, here we report $\xi \propto n^{1 / 2} \ell$ as the scaling parameter of $V_{\times}(G), R(G)$ and $H(G)$. This apparent mismatch can be understood by realizing that not only $\langle k\rangle$ but any function of it should scale end-vertex-degree based topological indices on ran- dom graphs; thus, since $\xi \propto\langle k\rangle^{1 / 2}$, for $\ell \ll 1$, our results agree with those in [49]. It is interesting to highlight, however, that the scaling parameter of the spectral and eigenvector properties of RGGs can not be written in terms of $\langle k\rangle$, as clearly shown in Table $\prod$ thus, validating the need of the scaling study developed in Sec. IIIB

We hope that our work may motivate further analytical as well as numerical studies on the applications of topological indices to random networks.

\section{ACKNOWLEDGMENTS}

J.A.M.-B. acknowledges financial support from FAPESP (Grant No. 2019/ 06931-2), Brazil, CONACyT (Grant No. 2019-000009-01EXTV-00067) and PRODEP-SEP (Grant No. 511-6/2019.-11821), Mexico. F.A.R. thanks CNPq (Grant No. 309266/2019-0) for the financial support given to this research.
[1] M. Barthélémy, Spatial networks, Phys. Rep. 499, 1 (2011).

[2] J. Dall and M. Christensen, Random geometric graphs, Phys. Rev. E 66, 016121 (2002).

[3] M. Penrose, Random Geometric Graphs (Oxford University Press, Oxford, 2003).

[4] E. N. Gilbert, Random plane networks, J. Soc. Ind. Appl. Math. 9, 533 (1961).

[5] M. D. Penrose, Connectivity of soft random geometric graphs, Ann. Appl. Probab. 26, 986 (2016).

[6] C. P. Dettmann and O. Georgiou, Random geometric graphs with general connection functions, Phys. Rev. E 93, 032313 (2016).

[7] E. Estrada and M. Sheerin, Random rectangular graphs, Phys. Rev. E 91, 042805 (2015).

[8] L. H. Wong, P. Pattison, and G. Robins, A spatial model for social networks, Physica A 360, 99 (2006).

[9] M. Shiino and T. Fukai, Self-consistent signal-to-noise analysis and its application to analogue neural networks with asymmetric connections, J. Phys. A Math. Gen. 25, L375 (1992).

[10] A. Diaz-Guilera, J. Gomez-Gardenes, Y. Moreno, and M. Nekovee, Synchronization in random geometric graphs, Int. J. Bifurc. Chaos 19, 687 (2009).

[11] J. Coon, C. P. Dettmann, and O. Georgiou, Impact of boundaries on fully connected random geometric networks, Phys. Rev. E 85, 011138 (2012).

[12] M. Nekovee, Worm epidemics in wireless ad hoc networks, New J. Phys. 9, 189 (2007).

[13] M. Haenggi, J. G. Andrews, F. Baccelli, O. Dousse, and M. Franceschetti, Stochastic geometry and random graphs for the analysis and design of wireless networks, IEEE J. Select. Areas Comm. 27, 1029 (2009).

[14] G. Mao and B. D. Anderson, Connectivity of large wireless networks under a general connection model, IEEE Trans. Inf. Theor. 59, 1761 (2013).

[15] C. P. Dettmann, O. Georgiou, and P. Pratt, Spatial net- works with wireless applications, Comptes Rendus Phys. 19, 187 (2018).

[16] Z. Toroczkai and H. Guclu, Proximity networks and epidemics, Physica A 378, 68 (2007).

[17] C. P. Brooks, J. Antonovics, and T. H. Keitt, Spatial and temporal heterogeneity explain disease dynamics in a spatially explicit network model, Am. Nat. 172, 149 (2008).

[18] P. Blackwell, M. Edmondson-Jones, and J. Jordan, Spectra of adjacency matrices of random geometric graphs, Research report: Dept. of Probability \& Statistics, University of Sheffield, no. 570/07, 2007. (http://www.jonathanjordan.staff.shef.ac.uk/rgg.html).

[19] A. Nyberg, T. Gross, and K. E. Bassler, Mesoscopic structures and the Laplacian spectra of random geometric graphs, J. Complex Networks 3, 543 (2015).

[20] C. P. Dettmann, O. Georgiou, and G. Knight, Spectral statistics of random geometric graphs, Europhys. Lett. 118, 18003 (2017).

[21] L. Alonso, J. A. Mendez-Bermudez, A. GonzalezMelendrez, and Y. Moreno, Weighted random-geometric and random-rectangular graphs: Spectral and eigenfunction properties of the adjacency matrix, J. Complex Networks 6, 753 (2018).

[22] J. A. Mendez-Bermudez, A. Alcazar-Lopez, A. J. Martinez-Mendoza, F. A. Rodrigues, and T. K. DM. Peron, Universality in the spectral and eigenfunction properties of random networks, Phys. Rev. E 91, 032122 (2015)

[23] L. Alonso, J. A. Mendez-Bermudez, and E. Estrada, Geometrical and spectral study of $\beta$-skeleton graphs, Phys. Rev. E 100, 062309 (2019).

[24] J. A. Mendez-Bermudez, G. Ferraz-de-Arruda, F. A. Rodrigues, and Y. Moreno. Scaling properties of multilayer random networks, Phys. Rev. E 96, 012307 (2017).

[25] C. T. Martinez-Martinez, J. A. Mendez-Bermudez, Y. Moreno, J. J. Pineda-Pineda, and J. M. Sigarreta. 
Spectral and localization properties of random bipartite graphs, Chaos Soliton Fract. X 3,100021 (2019).

[26] M. Randić, On characterization of molecular branching, J. Am. Chem. Soc. 97, 6609 (1975).

[27] I. Gutman and B. Furtula (Eds.), Recent results in the theory of Randić index, Univ. Kragujevac, Kragujevac, 2008.

[28] X. Li and I. Gutman, Mathematical aspects of Randić type molecular structure descriptors, Univ. Kragujevac, Kragujevac, 2006.

[29] X. Li and Y. Shi, A survey on the Randić index, MATCH Commun. Math. Comput. Chem. 59, 127 (2008).

[30] I. Gutman, B. Furtula, and V. Katanić, Randić index and information, AKCE Int. J. Graphs Comb. 15, 307 (2017).

[31] N. Nikolova and J. Jaworska, Approaches to measure chemical similarity - a review, QSAR Comb. Sci. 22, 1006 (2003).

[32] M. Randić, On the history of the connectivity index: from the connectivity index to the exact solution of the protein alignment problem, SAR QSAR Environ. Res. 26, 1 (2015).

[33] E. Estrada, Quantifying network heterogeneity, Phys Rev. E 82, 066102 (2010).

[34] P. de Meo, F. Messina, D. Rosaci, G. M. L. Sarné, and A. V. Vasilakos, Estimating graph robustness through the Randić index, IEEE Trans. Cybern. 48, 3232 (2018).

[35] Z. Chen, M. Dehmer, F. Emmert-Streib, and Y. Shi, Entropy of weighted graphs with Randić weights, Entropy 17, 3710 (2015).

[36] S. Fajtlowicz, On conjectures of Graffiti-II, Congr. Numer. 60, 187 (1987).

[37] H. Deng, S. Balachandran, S. K. Ayyaswamy, and Y. B. Venkatakrishnan, On the harmonic index and the chromatic number of a graph, Discrete Appl. Math. 161, 2740 (2013).

[38] J. M. Rodriguez and J. M. Sigarreta, New results on the harmonic index and its generalizations, MATCH Commun. Math. Comput. Chem. 78, 387 (2017).
[39] R. Wua, Z. Tanga, and H. Deng, A lower bound for the harmonic index of a graph with minimum degree at least two, Filomat 27, 51 (2013).

[40] L. Zhong, The harmonic index for graphs, Appl. Math. Lett. 25, 561 (2012).

[41] L. Zhong and K. Xu, Inequalities between vertex-degreebased topological Indices, MATCH Commun. Math. Comput. Chem. 71, 627 (2014).

[42] C. T. Martinez-Martinez, J. A. Mendez-Bermudez, J. M. Rodriguez, and J. M. Sigarreta, Computational and analytical studies of the harmonic index in Erdös-Rényi models, submitted (2020).

[43] C. T. Martinez-Martinez, J. A. Mendez-Bermudez, J. M. Rodriguez, and J. M. Sigarreta, Computational and analytical studies of the Randić index in Erdös-Rényi models, Appl. Math. Comput. 377, 125137 (2020)

[44] Y. Y. Atas, E. Bogomolny, O. Giraud, and G. Roux, Distribution of the ratio of consecutive level spacings in random matrix ensembles, Phys. Rev. Lett. 110, 084101 (2013).

[45] G. F. de Arruda, E. Cozzo, T. P. Peixoto, F. A. Rodrigues, and Y. Moreno, Disease localization in multilayer networks, Phys. Rev. X 7, 011014 (2017).

[46] G. F. de Arruda, J. A. Mendez-Bermudez, F. A. Rodrigues, and Y. Moreno, Universality of eigenvector delocalization and the nature of the SIS phase transition in multiplex networks, arXiv:2005.08074.

[47] D. A. Vega-Oliveros, J. A. Mendez-Bermudez, and F. A. Rodrigues, Multifractality in random networks with power-law decaying bond strengths, Phys. Rev. E 99, 042303 (2019).

[48] G. Torres-Vargas, R. Fossion, and J. A. MendezBermudez, Normal mode analysis of spectra of random networks, Physica A 545, 123298 (2020).

[49] R. Aguilar-Sanchez, I. F. Herrera-Gonzalez, J. A. Mendez-Bermudez, and J. M. Sigarreta Average properties of general indices on random networks, submitted (2020). 